\title{
Thermally-induced formation of Si wire array on an ultrathin (111) silicon-on-insulator substrate
}

\author{
Zainal A. Burhanudin, Ratno Nuryadi, Yasuhiko Ishikawa, and Michiharu Tabe ${ }^{a)}$ \\ Research Institute of Electronics, Shizuoka University, 3-5-1 Johoku, Hamamatsu 432-8011, Japan \\ Yukinori Ono \\ NTT Basic Research Laboratories, Nippon Telegraph and Telephone Corporation, 3-1 Morinosato \\ Wakamiya, Atsugi, Kanagawa 243-0198, Japan
}

(Received 3 May 2005; accepted 29 July 2005; published online 12 September 2005)

\begin{abstract}
We have found that a Si wire array is formed by thermal agglomeration of an ultrathin (111) Si layer in a bonded silicon-on-insulator (SOI) structure, although previous studies for crystalline and amorphous $\mathrm{Si}$ layers on $\mathrm{SiO}_{2}$ only showed island formation. As starting material, (111) bonded SOI wafers with the top Si layers thinned to 5-9 nm were used. The samples were then subjected to a thermal treatment at $950{ }^{\circ} \mathrm{C}$ in an ultrahigh vacuum. Atomic force microscopy revealed that the (111) top Si layer is deformed into three sets of wire arrays in the three equivalent $\langle 11 \overline{2}\rangle$ directions. It is also shown that the patterning of a Si layer leads to the wire array selectively formed in one of these three directions. (C) 2005 American Institute of Physics. [DOI: 10.1063/1.2053354]
\end{abstract}

Silicon-on-insulator (SOI) structures with the top $\mathrm{Si}$ layer as thin as $10 \mathrm{~nm}$ or below have attracted much interest in the formation of Si-based nanodevices. However, it has been shown that such ultrathin $\mathrm{Si}$ layers on $\mathrm{SiO}_{2}$ are thermally unstable, agglomerating into $\mathrm{Si}$ islands upon ultrahigh vacuum (UHV) annealing. ${ }^{1-8}$ The report on thermal agglomeration by Ono et al. ${ }^{1}$ showed that randomly formed $\mathrm{Si}$ islands are observed when an ultrathin Si layer of separationby-implanted-oxygen wafer is subjected to UHV annealing. Several other groups reported island formation for either crystalline or amorphous ultrathin $\mathrm{Si}$ layers on $\mathrm{SiO}_{2}{ }^{2-8} \mathrm{In}$ particular, our group reported an ordered formation of islands in the $\langle 310\rangle$ directions for the (001) Si layer of bonded SOI wafer. $^{5-7}$ This ordering property led us to further investigate the applicability to the quantum dot array through patterning of the Si layer. ${ }^{8}$ Nonetheless, as revealed in the present study, the agglomeration of Si layer does not necessarily result in deformation into islands. The present letter describes the deformation into a Si wire array of an ultrathin (111) Si layer of bonded SOI upon thermal agglomeration.

Samples used in the present work were prepared from (111) UNIBOND SOI wafers with a top Si layer of $305 \mathrm{~nm}$ and a buried oxide (BOX) layer of $400 \mathrm{~nm}$. The top Si layer was thinned to 5-9 $\mathrm{nm}$ by thermal oxidation followed by chemical etching of the grown oxide in a diluted $\mathrm{HF}$ solution. After treatment in a $\mathrm{H}_{2} \mathrm{SO}_{4}: \mathrm{H}_{2} \mathrm{O}_{2}$ mixture to form a $\sim 1 \mathrm{~nm}$-thick chemical oxide on the surface, the samples were placed in a UHV chamber at $6.0 \times 10^{-10}$ Torr and degassed for $2 \mathrm{~h}$ at $550{ }^{\circ} \mathrm{C}$. They were then annealed at $950{ }^{\circ} \mathrm{C}$ for $10 \mathrm{~s}$. Finally, the samples were retrieved from the UHV chamber and characterized using atomic force microscopy (AFM) and transmission electron microscopy (TEM).

Figures 1(a)-1(c) show typical AFM images, taken after annealing, of samples with an initial Si layer thicknesses of 9, 7, and $5 \mathrm{~nm}$, respectively. All three cases exhibited agglomeration of the Si layer. In the case of the $9 \mathrm{~nm}$-thick Si

\footnotetext{
a) Author to whom correspondence should be addressed; electronic mail:
} romtabe@rie.shizuoka.ac.jp
[Fig. 1(a)], the BOX surface (the darkest region at the center) is partially exposed, while the agglomerated $\mathrm{Si}$ (the bright lines) surrounds the exposed BOX surface. Upon reducing the Si thickness to $7 \mathrm{~nm}$ [Fig. 1(b)], the exposed BOX surface expands, leading to the formation of $\mathrm{Si}$ wires in the $\langle 11 \overline{2}\rangle$ directions. This wire formation is quite different from the island formation previously reported for crystalline and amorphous Si films on $\mathrm{SiO}_{2} \cdot{ }^{1-8}$ Upon further reduction of the Si thickness to $5 \mathrm{~nm}$, agglomeration occurs across the entire surface, forming an array of $\mathrm{Si}$ wires, as in Fig. 1(c). The average height, width, and spacing are 40,150, and $300 \mathrm{~nm}$, respectively. The total volume of $\mathrm{Si}$ wires is approximately the same as that of the initial Si layer, i.e., the evaporation of the $\mathrm{Si}$ atoms as $\mathrm{SiO}$ molecules is not dominant under this annealing condition, which is similar to the case of (001) Si layers. ${ }^{7}$ The observed thickness-dependent agglomeration is probably due to the strain in the Si layer. ${ }^{6}$ According to $\mathrm{Ca}-$ massel et al. ${ }^{9}$ the mismatch in thermal expansion coefficient between $\mathrm{Si}\left(2.6 \times 10^{-6} \mathrm{~K}\right)$ and $\mathrm{SiO}_{2}\left(0.5 \times 10^{-6} \mathrm{~K}\right)$ induces a strain in the $\mathrm{Si}$ layer, which increases with decreasing top $\mathrm{Si}$ thickness. In order to release the strain energy, thinner $\mathrm{Si}$ layers with higher strains may have resulted in the wider area of the agglomerated region.

The AFM image in Fig. 1(c) suggests that the wires in the $[\overline{1} 2 \overline{1}]$ direction are more stable than those in the direc-

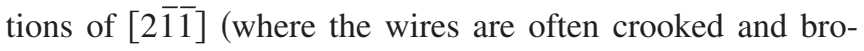
ken) and [1 12$]$ (where the wires are usually missing and replaced by voids), although these three directions should be equivalent. This asymmetric wire formation could be due to the atomic steps on the initial top Si surface, which are derived from the slight miscut from the (111) plane. As in the AFM image of Fig. 2, which was taken on the top Si surface in the nonagglomerated region, the atomic steps run in a direction almost perpendicular to the $[\overline{1} 2 \overline{1}]$ direction. The former is the direction along which the wire array was preferably formed. Taking into account the restrictive terrace-toterrace diffusion of surface atoms due to the Schwoebel barrier, ${ }^{10}$ it is reasonable that the wires were preferentially 

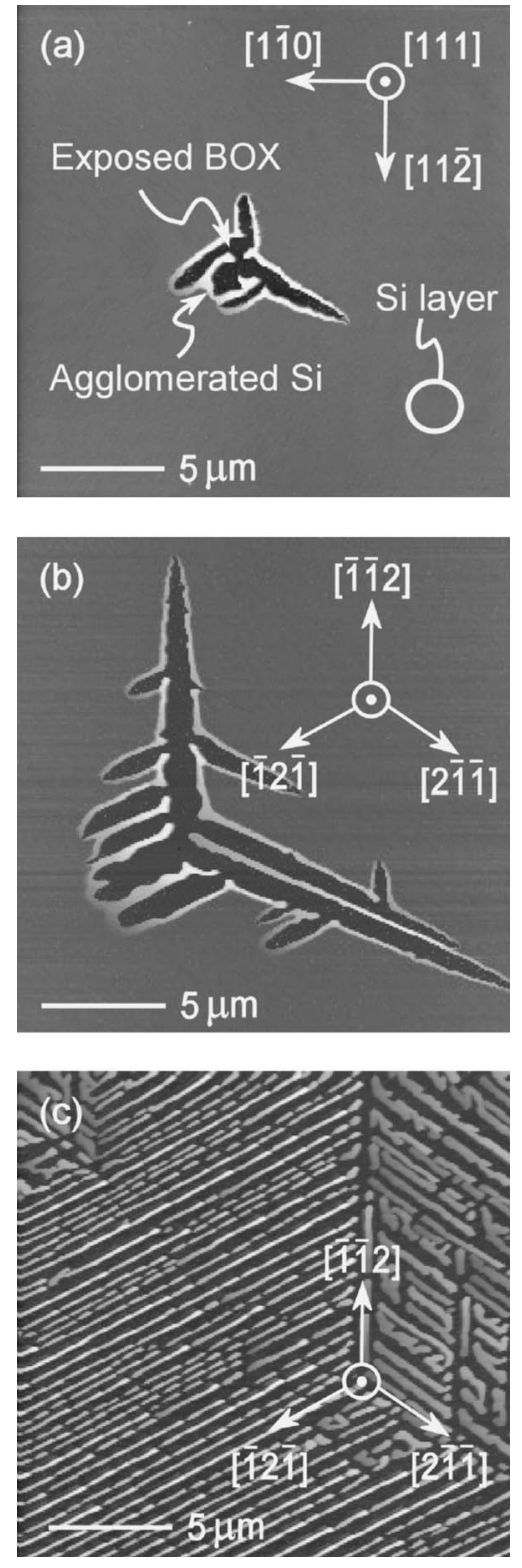

FIG. 1. AFM images taken after UHV annealing of (111) Si layers. The initial thicknesses were (a) $9 \mathrm{~nm}$, (b) $7 \mathrm{~nm}$, and (c) $5 \mathrm{~nm}$.

formed normal to the atomic steps, i.e., along the $[\overline{1} 2 \overline{1}] \mathrm{di}-$ rection.

In order to examine the facet structures on the wire surface, which are presumably responsible for the wire formation, cross-sectional TEM observations were carried out. Figure 3(a) shows a typical TEM image of a wire running along the $[\overline{1} 2 \overline{1}]$ direction, and Fig. 3(b) shows the left side of the wire at a high magnification. As shown in Fig. 3(b), the wire is surrounded by $(31 \overline{1})$ and $(10 \overline{1})$ facets on the sidewall, separated by two intermediate $(531)$ and $(81 \overline{6})$ facets, in addition to the top (111) plane. ${ }^{11}$ The same facet structures were observed on the right side, as well as for the wires running along the $[\overline{1} \overline{1} 2]$ and $[2 \overline{1} \overline{1}]$ directions. We believe that the observed facet planes are thermally stable, although it is not clear whether or not the two intermediate facets with high indices have low surface energies. ${ }^{12}$ For the agglomeration of the Si layer, the $\{311\}$ group facets should be particularly important since these facets play a significant role in ordered island formation along the $\langle 310\rangle$ directions in the

Downloaded $10 \mathrm{Jul} 2008$ to 133.70 .80 .50 . Redistribution subject to $A$

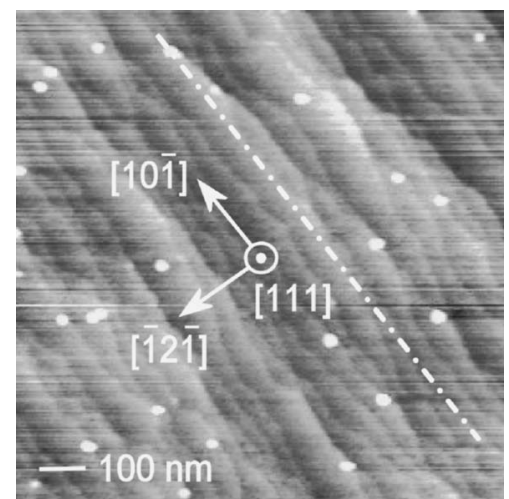

FIG. 2. An AFM image showing the atomic steps on the (111) Si surface. The steps run almost normal to the $[\overline{1} 2 \overline{1}]$ direction.

case of the (001) Si layer. ${ }^{5-8}$ In the case of (111) Si layer, such $\{311\}$ facets can appear as sidewalls on both the left and right sides in the $\langle 11 \overline{2}\rangle$ cross-section (as described above), while in the $\langle 1 \overline{1} 0\rangle$ cross-section, the $\{311\}$ facet can appear as the sidewall only on one side due to the asymmetric atomic arrangement. This asymmetry property in $\{311\}$ facets is presumably responsible for the formation of wires, rather than islands. However, the one-sided $\{311\}$ facets in the $\langle 1 \overline{1} 0\rangle$ cross-section may cut the wires running along the $\langle 11 \overline{2}\rangle$ directions, resulting in the short wires seen in Fig. 1(c).

It would be useful to form wires aligned in only one of the three $\langle 11 \overline{2}\rangle$ directions for practical applications such as $\mathrm{Si}$ wire transistors. As an attempt, patterning of the Si layer was investigated. An example of an AFM image taken near the edge of a pattern is shown in Fig. 4. In this case, the pattern edge runs along the $[11 \overline{2}]$ direction, with the Si layer on its left side before annealing. It is found that this pattern forms wires only in the $[\overline{1} \overline{1} \overline{1}]$ direction. This mono-directional wire formation was also achieved with patterns along other directions, although the alignment direction selected from the three equivalent $\langle 11 \overline{2}\rangle$ directions depended on the pattern
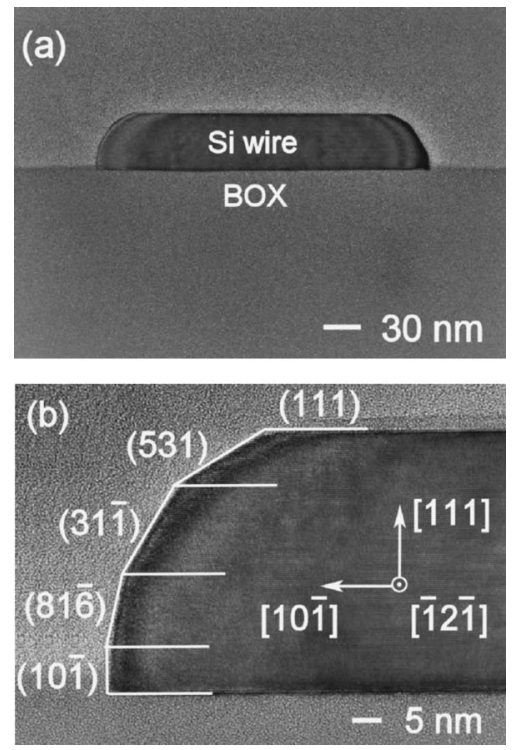

FIG. 3. Cross-sectional TEM images of a wire: (a) an overall view, (b) a

higher-magnification image with facet assignments.

AIP license or copyright; see http://apl.aip.org/apl/copyright.jsp 


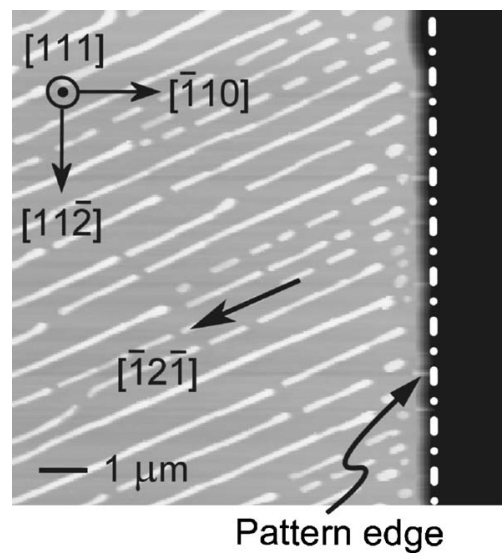

FIG. 4. An AFM image taken at the pattern edge of the (111) Si layer.

direction. Thus, the alignment direction of wires can clearly be selected via patterning of the $\mathrm{Si}$ layer. The monodirectional behavior can be ascribed again to the $\{311\}$ facets, which are seen at the edge of the wires. Figure 5(a) shows an example of an AFM image taken at the edge of a wire selec-

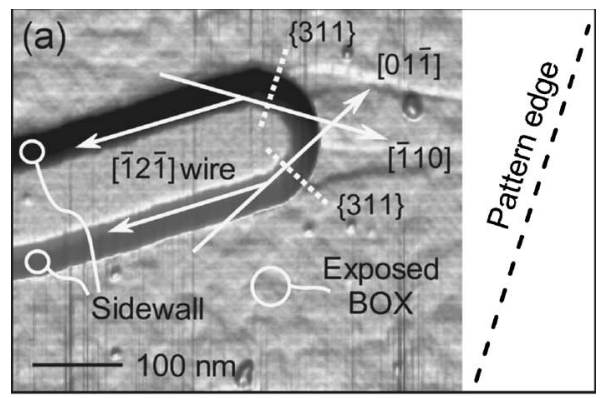

(b)

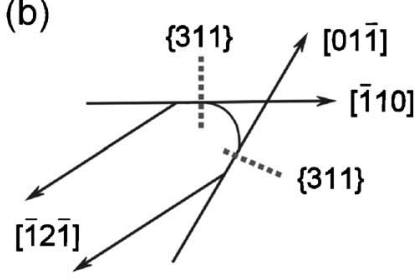

Actual wire

(c)

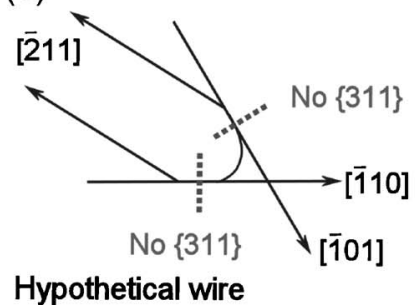

FIG. 5. (a) An AFM image taken at the edge of a wire in the [1릴 direction. The observed wire in (a) and the hypothetical wire (discussed in the main text) are schematically shown in (b) and (c), respectively. tively formed in the $[\overline{1} 2 \overline{1}]$ direction. The line profiles taken along the dashed lines revealed that the $\{311\}$ facet planes are present at the edge of the wire as sidewalls along the [110] and $[01 \overline{1}]$ directions. In contrast to the situation in Fig. 5(a) [schematically shown in Fig. 5(b)], if the wire is formed in the $[\overline{2} 11]$ direction, as assumed in Fig. 5(c), the $\{311\}$ sidewalls along the $\langle\overline{1} 10\rangle$ direction cannot appear at the edge of the wire because of the asymmetric atomic arrangement in the $\langle\overline{1} 10\rangle$ direction. Taking this property into account, it is not surprising that mono-directional alignment can be realized via patterning. Of course, for large-sized patterns, agglomeration may occur not only from the pattern edge, but also from the inside of the pattern, as in the case of unpatterned $\mathrm{Si}$ shown in Fig. 1. Since structural singularities, such as small protrusions and holes on the BOX surface as well as the thinner section in the top Si layer, would initiate agglomeration in the unpatterned SOI, ${ }^{6-8}$ the removal of such singularities presumably leads to mono-directional wire formation even for large sized patterns.

In summary, we demonstrated that thermal agglomeration of the (111) Si layer of a bonded SOI wafer yields a $\mathrm{Si}$ wire array, in contrast to the island formation previously reported for crystalline and amorphous $\mathrm{Si}$ layers on $\mathrm{SiO}_{2}$. The wires were aligned in the three equivalent $\langle 11 \overline{2}\rangle$ directions, but patterning enabled the selection of one of these three as the alignment direction.

This work was partly supported by a Grant-in-Aid for Scientific Research from the Japan Society for the Promotion of Science.

${ }^{1}$ Y. Ono, M. Nagase, M. Tabe, and Y. Takahashi, Jpn. J. Appl. Phys., Part 1 34, 1728 (1995)

${ }^{2}$ N. Sugiyama, T. Tezuka, and A. Kurobe, J. Cryst. Growth 192, 395 (1998).

${ }^{3}$ Y. Wakayama, T. Tagami, and S. Tanaka, J. Appl. Phys. 85, 8492 (1999).

${ }^{4}$ B. Legrand, V. Agache, T. Melin, J. P. Nys, V. Senez, and D. Stievenard, J. Appl. Phys. 91, 106 (2002)

${ }^{5}$ R. Nuryadi, Y. Ishikawa, and M. Tabe, Appl. Surf. Sci. 159, 121 (2000).

${ }^{6}$ R. Nuryadi, Y. Ishikawa, Y. Ono, and M. Tabe, J. Vac. Sci. Technol. B 20, 167 (2002).

${ }^{7}$ Y. Ishikawa, M. Kumezawa, R. Nuryadi, and M. Tabe, Appl. Surf. Sci. 190, 11 (2002).

${ }^{8}$ Y. Ishikawa, Y. Imai, H. Ikeda, and M. Tabe, Appl. Phys. Lett. 83, 3162 (2003).

${ }^{9}$ J. Camassel, L. A. Falkovsky, and N. Planes, Phys. Rev. B 63, 035309 (2001).

${ }^{10}$ T. T. Tsong, Prog. Surf. Sci. 64, 199 (2000).

${ }^{11}$ The detailed TEM observation showed that the bottom of the Si wire near the sidewalls is slightly depressed into the underlying $\mathrm{SiO}_{2}$. This is probably due to the evaporation of slight amount of $\mathrm{Si}$ atoms as $\mathrm{SiO}$ molecules, although such a phenomenon is difficult to be detected by the volume analysis based on the AFM observation.

${ }^{12}$ D. J. Eaglesham, A. E. White, L. C. Feldman, N. Moriya, and D. C. Jacobson, Phys. Rev. Lett. 70, 1643 (1993). 